\title{
Comparison of short-term and survival outcomes for transanal natural orifice specimen extraction with conventional mini-laparotomy after laparoscopic anterior resection for colorectal
} cancer

\author{
Sicheng Zhou* \\ Xuewei Wang* \\ Chuanduo Zhao \\ Wei Pei \\ Haitao Zhou \\ Qian Liu \\ Jianwei Liang \\ Zhixiang Zhou \\ Xishan Wang
}

Department of Colorectal Surgery, National Cancer Center/National Clinical Research Center for Cancer/Cancer Hospital, Chinese Academy of Medical Sciences and Peking Union Medical College, Beijing 10002I, People's Republic of China

*These authors contributed equally to this work
This article was published in the following Dove Press journal: Cancer Management and Research

Background: Transanal natural orifice specimen extraction (NOSE) in colorectal surgery has been introduced as a less invasive surgery. However, its long-term survival effects remain controversial. The aim of this study was to compare the short-term and long-term survival outcomes of transanal NOSE with those of the conventional laparoscopic approach with mini-laparotomy (LAP) for anastomosis construction and specimen extraction in sigmoid colon cancer or rectal cancer.

Methods: From January 2007 to January 2018, a retrospective study was conducted at the China National Cancer Center. In total, 52 consecutive patients who underwent laparoscopic anterior resection with NOSE were matched with an additional 52 patients who underwent conventional LAP for colorectal cancer.

Results: Patients in the NOSE group experienced shorter time to passage of flatus $(2.8 \pm 0.8$ vs $3.2 \pm 0.9$ days; $p=0.042)$, less pain $(4.2 \pm 1.4$ vs $5.4 \pm 1.7 ; p=0.003)$ and less analgesia required $(7.7 \%$ vs $25.0 \%$; $p=0.032$ ). After a median follow-up of 68.5 (range, $8-83$ ) months, the two groups had similar 5-year overall survival rates $(92.3 \%$ vs $94.2 \% ; p=0.985)$ and disease-free survival rates $(84.6 \%$ vs $86.5 \% ; p=0.802)$.

Conclusion: Transanal NOSE for total laparoscopic anterior resection is safe and feasible with more advantages, including lower pain, lower tissue trauma and faster recovery of intestinal function. Moreover, with proper protection of the surgical route, transanal NOSE has the same long-term outcomes as conventional laparoscopic surgery.

Keywords: natural orifice specimen extraction, transanal specimen extraction, total laparoscopic resection, sigmoid colon cancer, rectal cancer, surgical outcomes

\section{Introduction}

In recent decades, techniques and instrumentation for laparoscopic surgery have been evolving in an effort to decrease the number and the size of incisions. ${ }^{1,2}$ However, a small muscle-split incision is made during colorectal laparoscopic resection to extract the specimen and to create the proximal part of the bowel anastomosis. Advanced technology and accumulated experience in laparoscopic surgery have prompted surgeons to find less invasive methods than multiport laparoscopic surgery. Some reports have described total intracorporeal anastomosis
Correspondence: Jianwei Liang National Cancer Center/National Clinical Research Center for Cancer/Cancer Hospital, Chinese Academy of Medical Sciences and Peking Union Medical College, Beijing 10002I, People's Republic of China Tel +86I 76I06I I585

Email Liangjw1976@I63.com 
combined with extraction of the specimen through natural orifices, such as the anus and the vagina. ${ }^{3-5}$ The concept of natural orifice specimen extraction (NOSE) is not new. This approach can eliminate a long $(5-10 \mathrm{~cm})$ abdominal incision other than that for trocar placement. NOSE gained attention due to its reduced abdominal incision, better cosmesis and its theoretical subsequently decreased rates of wound infection, postoperative pain and surgical stress response. ${ }^{6,7}$ Transanal extraction is a valid option for leftsided colectomy, rectosigmoid resection or restorative proctocolectomy. Since Franklin et $\mathrm{al}^{8}$ reported a series of patients who underwent laparoscopic sigmoid colon resection with transanal specimen removal in 1993, there have been a number of case series reporting laparoscopic colectomy with NOSE. However, most of these studies were limited by small numbers. ${ }^{9-11}$ Moreover, very few studies compared the clinical outcomes of NOSE with conventional laparoscopic colectomy, especially longterm survival outcomes. ${ }^{12,13}$

Transanal specimen extraction has been applied in laparoscopic anterior resection for rectal cancer and sigmoid colon cancer in our institution since 2012. Therefore, we conducted a single center, case-matched analysis to compare short-term and long-term survival outcomes in patients who underwent laparoscopic anterior resection with intracorporeal anastomosis and transanal specimen extraction with patients who underwent conventional laparoscopic anterior resection with mini-laparotomy.

\section{Materials and methods}

From January 2012 to December 2013, a consecutive series of patients who had an indication for laparoscopic anterior resection or low anterior resection for rectal cancer and sigmoid colon cancer were invited to participate. Patients who underwent total laparoscopic resection with intracorporeal anastomosis and transanal specimen extraction were assigned to the NOSE group, and patients who received conventional laparoscopic resection with mini-laparotomy were assigned to the LAP group. The indications for total laparoscopic anterior resection with transanal NOSE were as follows: patients were diagnosed of sigmoid colon cancer or rectal cancer, 18 to 75 years old, distance of tumor from the anal verge was $6-30 \mathrm{~cm}$, tumor size $<5.0 \mathrm{~cm}$ and BMI (body mass index) $\leq 28$. Based on this surgical registry, a group of 55 patients who underwent total laparoscopic anterior or low anterior resection with transanal NOSE was identified. Transanal access was not created during surgery in three of the 55 patients due to unexpected findings (bulky tumor in one patient, mesentery fat in another patient and narrow pelvis in the third patient), and we switched to conventional laparoscopic-assisted resection. These 52 consecutive procedures were individually matched with 52 conventional laparoscopically assisted resection procedures by an independent reviewer, who was blinded to all clinical data, scrutinized consecutively from 2012 backwards. Matching criteria included patient age ( \pm 2 years), gender, body mass index (BMI), date of surgery ( -1 year), American Society of Anaesthesiologists (ASA) score, comorbidity, previous abdominal surgery, tumor location, clinical tumor (T) category and preoperative chemoradiotherapy.

All patients underwent a curative resection by one experienced surgeon, and all enrolled patients underwent radical surgery in accordance with the total mesorectal excision (TME) principle. The American Joint Committee on Cancer (AJCC, the eighth edition) staging system was used for tumor staging. The guidelines of the National Comprehensive Cancer Network (NCCN) were used for perioperative management. All patients received preoperative assessment, including physical and laboratory examinations, colonoscopy with biopsy, abdominal computed tomography scan and pelvic magnetic resonance imaging. Patients with T3, T4 or N+ mid- or low rectal cancer received preoperative chemoradiotherapy followed by surgery 6 weeks later. In postoperative care, patient-controlled analgesia (PCA) was applied for all patients, and additional intravenous analgesia was administered when required. Sips of water began when patients had no discomfort in the abdomen after first flatus, and a soft diet was started one day thereafter. Routine laboratory tests and CRP levels were measured on the day before surgery and on postoperative days 1, 3 and 5. All patients signed the written informed consent and complied with the Declaration of Helsinki. This study was approved by the ethics committee of Cancer Hospital, Chinese Academy of Medical Sciences (NCC 2017-YZ-026, Oct 17, 2017)

\section{Surgical technique}

The patient was placed in modified lithotomy, trendelenburg position with a pneumoperitoneum of $12 \mathrm{mmHg}$. Four trocars $(2 \times 12 \mathrm{~mm}$ and $2 \times 0.5 \mathrm{~mm})$ were used in most cases. The camera trocar was placed supraumbilically, and two trocars were placed on the right side in the middle and lower abdomen in line with the spina iliaca anterior superior. The fourth trocar was positioned in the left lower abdomen. Laparoscopic skill was applied according to radical principle. High ligation of the inferior 
mesenteric vessel, mobilization of the bowel and dissection of the lymph nodes were performed laparoscopically, and total mesorectal excision with nerve-sparing techniques was followed for rectal cancer. Then, different procedures were performed for the laparoscopic-assisted approach and NOSE.

We previously reported the technical aspects of standard laparoscopic NOSE for sigmoid colon and rectum carcinoma. ${ }^{14,15}$ Briefly, for the NOSE technique, a cross clamp was placed distal to the tumor after adequate mobilization of the sigmoid colon or rectum to ensure the segment bearing tumor was isolated. For sigmoid colon cancer, the clamp position was $10-12 \mathrm{~cm}$ from the anal verge. For rectal cancer, the clamp placed was $1.5-2.0 \mathrm{~cm}$ from the tumor. The distal rectum was transected by ultrasonic scalpel after fully disinfecting the rectal lumen by $10 \%$ povidone-iodine. An anvil head attached to circular stapling device was placed into the abdominal cavity through the anus and rectal stump. A longitudinal incision approximately $2 \mathrm{~cm}$ was generated on proximal colon wall, and the anvil of circular stapling device was inserted into the colon lumen through the incision. Then, the proximal colon was transected in close proximity to the upper pole of the incision using a linear stapling device. The trocar in the right lower quadrant was removed, and a soft tissue retractor (Product Model: HK-120/130-120/100) was inserted into the abdominal cavity via the wound. One of a pair of the soft tissue retractor rings was held and pulled out of the anus, and another ring was placed in the opened rectal stump. A long Babcock grasper was inserted transanally, and the specimen was extracted through the soft tissue retractor. Then, the soft tissue retractor was removed. In the later 28 cases, we used the disposable sterile protective cover (Icare Medical Co., LTD, 20×40 cm) instead of the soft tissue retractor. The rectal opening was reclosed using an endoscopic linear stapler, and the additional section of rectal wall was placed into a specimen bag and extracted through the port in right lower quadrant. End-to-end colorectal anastomosis was performed with a circular stapler using the double-stapling technique.

For conventional laparoscopic-assisted surgery, a small incision $3-7 \mathrm{~cm}$ in length was made in the hypogastrium, and transection of the rectum was completed through disposable incision dilator. Then, the specimen was removed, and the bowel was prepared for anastomosis. The anastomosis for all rectal cancer cases and most of the sigmoid colon cancer cases was performed by a double-stapling technique following open resection. For some sigmoid colon cancer cases for which the tumor site was comparatively higher, three straight line-cutting devices were selected for anastomosis.

\section{Follow-up}

According to the guidelines of the $\mathrm{NCCN}$, all patients with T3/T4 or postoperative node-positive tumors underwent adjuvant chemotherapy postoperatively. All patients were scheduled to receive follow-up through outpatient visits every 3-6 months in the first three years. Physical and laboratory examinations, including tumor biomarkers (CEA and CA-199), were performed at every follow-up. Every half year, CT scans of the chest, abdomen and pelvis were performed, and a complete colonoscopy was planned for each year. Three years after surgery, the patients were followed up every 6-12 months by outpatient visits or telephone until death due to recurrence and metastasis of CRC or December 31, 2018, whichever came first. The long-term endpoints of this study were 5-year overall survival (OS) and disease-free survival (DFS).

\section{Statistical analysis}

Data analysis was performed using SPSS for Windows version 20.0 (SPSS, Chicago, Illinois, USA). Betweengroup differences in continuous data were analyzed using Student's $t$-test and Mann-Whitney $U$ test for independent values for normally and non-normally distributed values, respectively. Categorical data were compared by means of $\chi^{2}$ test or Fisher's exact test, as appropriate. The KaplanMeier method was performed to calculate the survival outcomes of the patients in the two groups, and the differences of the survival curves (OS and DFS) were compared by a log-rank test. Statistical significance was established as $p<0.05$.

\section{Results}

\section{Clinical characteristics}

Between January 2012 and December 2013, a total of 224 consecutive patients with sigmoid colon or rectal cancer underwent a laparoscopic anterior resection or low anterior resection by Professor Zhou. The preoperative clinical characteristics of the 52 patients in the NOSE group and 52 matched control cases in LAP group are shown in Table 1. After matching, the clinical characteristics, including age, gender, BMI, ASA category, comorbidity, previous abdominal surgery, tumor location, clinical $\mathrm{T}$ category and preoperative chemoradiotherapy, were well balanced between 
Table I Patient characteristics

\begin{tabular}{|c|c|c|c|}
\hline Characteristic & NOSE $(n=52)$ & $\operatorname{LAP}(n=52)$ & p-value \\
\hline Age, year (mean $\pm S D)$ & $55.6 \pm 10.4$ & $57.0 \pm 10.7$ & 0.468 \\
\hline Gender & & & 1.000 \\
\hline Male & 27 & 27 & \\
\hline Female & 25 & 25 & \\
\hline $\mathrm{BMI}, \mathrm{kg} / \mathrm{m}^{2}($ mean $\pm \mathrm{SD})$ & $22.7 \pm 2.7$ & $23.1 \pm 2.9$ & 0.367 \\
\hline ASA & & & 0.285 \\
\hline I & 12 & 6 & \\
\hline II & 38 & 43 & \\
\hline III & 2 & 3 & \\
\hline Preoperative comorbidity & & & 0.395 \\
\hline Presence & 14 & 18 & \\
\hline Absence & 38 & 34 & \\
\hline Abdominal operation history & & & 0.300 \\
\hline Presence & 11 & 7 & \\
\hline Absence & 41 & 45 & \\
\hline Tumor size, $\mathrm{cm}($ mean $\pm \mathrm{SD})$ & $3.4 \pm 1.3$ & $3.7 \pm 1.0$ & 0.142 \\
\hline Distance of tumor from anal verge, $\mathrm{cm}($ mean $\pm \mathrm{SD})$ & $14.5 \pm 7.5$ & $|5| \pm 8.4$. & 0.703 \\
\hline Tumor location & & & $0.84 I$ \\
\hline Sigmoid colon & 21 & 20 & \\
\hline Rectum & 31 & 32 & \\
\hline Preoperative serum CEA $(\mathrm{ng} / \mathrm{mL})($ mean $\pm \mathrm{SD})$ & $4.3 \pm 6.3$ & $6.2 \pm 8.5$ & 0.186 \\
\hline Preoperative serum CAI9-9 $(\mathrm{ng} / \mathrm{mL})($ mean \pm SD) & $16.3 \pm 20.6$ & $16.2 \pm 19.5$ & 0.987 \\
\hline Incidence of preoperative CEA elevation & $10(19.2)$ & $17(32.7)$ & 0.117 \\
\hline Incidence of preoperative CAI9-9 elevation & $4(7.7)$ & $4(7.7)$ & 1.000 \\
\hline Clinical tumor category & & & 0.234 \\
\hline $\mathrm{TI}$ & 11 & 5 & \\
\hline $\mathrm{T} 2$ & 7 & 10 & \\
\hline T3 & 34 & 37 & \\
\hline Preoperative chemoradiotherapy & 8 & 10 & 0.604 \\
\hline
\end{tabular}

Note: NOSE, totally laparoscopic anterior resection with transanal specimen extraction.

Abbreviations: LAP, laparoscopic anterior resection with minilaparotomy; BMI, body mex index; CEA serum carcinoembryonic antigen; CAI9-9, carbohydrate antigen I9-9.

the two groups. Furthermore, the two groups were similar in terms of tumor size, distance of tumor from anal verge and preoperative serum tumor markers (all $p<0.05$ ).

\section{Short-term outcomes}

Intraoperative and postoperative outcomes are summarized in Table 2. Patients in both groups underwent a similar type of operation. The mean duration of surgery was essentially identical (NOSE 111.2 $\pm 23.5 \mathrm{~min}$ vs LAP $110.9 \pm 23.6 \mathrm{~min}$; $p=0.963$ ). No conversion to open surgery was needed in the two groups, and no intraoperative complications were encountered in either group. An early indicator of postoperative recovery, the time to first flatus, was significantly shorter in the NOSE group (2.8 \pm 0.8 vs $3.2 \pm 0.9$ days; $p=0.042)$. Although patients in the NOSE group showed better results than the LAP group in terms of time to regular diet and length of hospital stay after surgery, the difference was not statistically significant. Patients in the NOSE group experienced significantly less postoperative pain on day 1 after surgery than those in LAP group [visual analog scale (VAS) score: $4.2 \pm 1.4$ vs $5.4 \pm 1.7, p=0.003]$. Fewer patients in the NOSE group required additional rescue analgesia or parenteral analgesia (4 (7.7\%) vs $13(25.0 \%) ; p=0.032)$. Postoperative complications occurred in four patients in the NOSE group and in eight patients in the conventional laparoscopically assisted procedure. Complications in the 
Table 2 Operative and postoperative outcomes

\begin{tabular}{|c|c|c|c|}
\hline Parameter & NOSE $(n=52)$ & LAP $(n=52)$ & p-value \\
\hline Type of operation, case (\%) & & & 1.000 \\
\hline Anterior resection & $30(57.7)$ & $30(57.7)$ & \\
\hline Low anterior resection & $22(42.3)$ & $22(42.3)$ & \\
\hline Operative time, mins (mean $\pm \mathrm{SD}$ ) & $111.2 \pm 23.5$ & $110.9 \pm 23.6$ & 0.963 \\
\hline Estimated blood loss, $\mathrm{mL}$ (mean $\pm \mathrm{SD}$ ) & $49.8 \pm 48.1$ & $62.5 \pm 47.1$ & 0.177 \\
\hline Time to first flatus, day (mean $\pm S D$ ) & $2.8 \pm 0.8$ & $3.2 \pm 0.9$ & 0.042 \\
\hline Time to Regular diet, day (mean $\pm S D$ ) & $3.9 \pm 0.6$ & $4.1 \pm 0.9$ & 0.068 \\
\hline Postoperative hospital stay, day (mean \pm SD) & $9.0 \pm 2.0$ & $9.4 \pm 3.2$ & 0.380 \\
\hline Post-operative complication, case (\%) & $4(7.7)$ & $8(15.4)$ & 0.358 \\
\hline Anastomotic leakage & I & I & \\
\hline Wound infection & 0 & 2 & \\
\hline Haemorrhage requiring transfusion & 2 & 1 & \\
\hline Intra-abdominal abscess & 1 & 1 & \\
\hline Pulmonary infection & 0 & I & \\
\hline Urinary retention & 0 & I & \\
\hline lleus & 0 & I & \\
\hline Reoperation, case (\%) & 0 & I (I.9) & \\
\hline VAS (PODI), (mean \pm SD) & $4.2 \pm 1.4$ & $5.4 \pm 1.7$ & 0.003 \\
\hline Additional analgesia required, case (\%) & $4(7.7)$ & $13(25.0)$ & 0.032 \\
\hline Death & 0 & 0 & \\
\hline Leukocytosis (PODI), case (\%) & $40(76.9)$ & $32(61.5)$ & 0.089 \\
\hline WBC count difference (PODI), $\left(\times 10^{9}\right)$ & $11.8 \pm 2.5$ & $11.6 \pm 3.5$ & 0.720 \\
\hline \multicolumn{4}{|l|}{ CRP } \\
\hline PODI, mg/dl (mean \pm SD) & $3.8 \pm 3.2$ & $2.3 \pm 0.9$ & 0.048 \\
\hline POD3, mg/dl (mean \pm SD) & $4.8 \pm 2.9$ & $9.4 \pm 5.9$ & 0.020 \\
\hline $\mathrm{POD} 5, \mathrm{mg} / \mathrm{dl}($ mean $\pm \mathrm{SD})$ & $1.7 \pm 1.9$ & $3.6 \pm 3.9$ & 0.153 \\
\hline
\end{tabular}

Note: NOSE, totally laparoscopic anterior resection with transanal specimen extraction.

Abbreviations: LAP, laparoscopic anterior resection with minilaparotomy; VAS, visual analogue scale.

NOSE group included postoperative bleeding in two patients, which required transfusion, and an intra-abdominal abscess, which was treated by percutaneous drainage and antibiotics without anastomotic leakage. One patient in the NOSE group experienced anastomotic leakage and was treated by conservative drainage, antibiotics and no oral intake without the creation of stoma. None of the patients in the NOSE group encountered complications associated with the extraction site, such as perineal pain or anal incontinence. Two patients in the LAP group developed wound infections at the assistant abdominal resection. Re-operation with ileostomy was performed in one patient who developed anastomotic leakage. Other complications in the LAP group included postoperative hemorrhage requiring transfusion in one patient, intro-abdominal abscess recovered with drainage in one patient, pneumonia in one patient, urinary retention in one patient and an ileus. No deaths were recorded during the perioperative period in either group.
With respect to the laboratory examination, the incidence of immediate postoperative leucocytosis in the NOSE group (76.9\%) was greater compared with the LAP group (61.5\%), but the difference was not statistically significant $(p=0.089)$. No significant differences were noted between the two groups with respect to leukocyte count $(p=0.720)$. There was a significantly higher CRP level in the NOSE group one day after surgery than in the LAP group $(3.8 \pm 3.2$ vs $2.3 \pm 0.9 ; p=0.048)$. However, the mean CPR values on postoperative day 3 were significantly lower in the NOSE group compared with the LAP group ( $4.8 \pm 2.9$ vs $9.4 \pm 5.9 ; p=0.020)$.

The results of pathological evaluation of the surgical specimens are presented in Table 3. Mean tumor size and the length of the resection margin, including the proximal and distal margin, were similar between the groups. No patients had a positive resection margin. The mean number of harvested lymph nodes was $19.1 \pm 7.0$ in the NOSE 
Table 3 Pathological outcomes

\begin{tabular}{|l|l|l|l|}
\hline Parameter & NOSE (n=52) & LAP (n=52) & p-value \\
\hline Length of the specimen, cm (mean \pm SD) & $15.3 \pm 5.4$ & $15.5 \pm 4.8$ & 0.845 \\
Proximal margin, cm (mean \pm SD) & $12.2 \pm 8.7$ & $11.7 \pm 8.1$ & 0.544 \\
Distal margin, cm (mean \pm SD) & $3.9 \pm 1.6$ & $4.3 \pm 2.2$ & 0.310 \\
\hline TNM stage & & 13 & 0.409 \\
I & 16 & 17 & \\
II & 11 & 22 & \\
III & 25 & $19.8 \pm 8.2$ \\
\hline No. of lymph nodes retrieved (mean \pm SD) & $19.1 \pm 7.0$ & & \\
\hline Histological differentiation & & 7 & \\
Well & 13 & 41 & \\
Moderate & 37 & 4 & 0.263 \\
Poor & 2 & 7 & \\
\hline Perineural invasion & 8 & 4 & \\
Venous invasion & 2 & & \\
\hline
\end{tabular}

Note: NOSE, totally laparoscopic anterior resection with transanal specimen extraction.

Abbreviation: LAP, laparoscopic anterior resection with minilaparotomy.

group and 19.8 \pm 8.2 in the LAP group $(p=0.609)$. The distribution of histological differentiation and the tumornode-metastasis stages were also similar in the two groups.

\section{Survival analysis}

The median follow-up period was 68.5 (range, 8-83) months. During the whole follow-up period, 8 of the 104 patients died, and 15 patients had local recurrence or distant metastasis. There was no significant difference in tumor recurrence between the NOSE group and the LAP group. Two patients developed local recurrence and 6 patients developed distant recurrence in the NOSE group after a median follow-up of 68 (range, 30-81) months. One patient developed local recurrence and 6 patients developed distant recurrence in the LAP group after a median follow-up of 69.5 (range, 8-83) months (Table 4). No transanal accesssite or port-site recurrence occurred in either group. In addition, 2 patients in the NOSE group died from multiple hepatic recurrences at either 30 months or 40 months. One patient died from lung metastasis at 48 months, and one patient died from peritoneal cavity metastasis at 28 months. Meanwhile, three patients in the LAP group died at either 8 months, 52 months or 70 months after multiple hepatic recurrences, and one patient died at 28 months after peritoneal cavity metastasis. The Kaplan curves showed that the NOSE group had similar OS $(p=0.985)$ and DFS $(p=0.802)$ compared with the LAP group. The 5-year OS rate and DFS rate in the NOSE group were $92.3 \%$ and $84.6 \%$, respectively, and those in the LAP group were $94.2 \%$ and $86.5 \%$, respectively (Figures 1 and 2 ).

\section{Discussion}

The present study compared the short-term and long-term survival outcomes between sigmoid colon or rectal cancer patients who underwent NOSE and the patients who underwent conventional LAP using a case-matched design. Our results suggested that NOSE surgery may be safe and acceptable with better short-term outcomes, such as improved postoperative recovery, less pain and lower trauma, compared with the LAP group. Furthermore, the OS and DFS of NOSE group were similar to that of LAP group.

Previous studies demonstrated that patients who underwent anterior resection with transanal NOSE for sigmoid or rectal cancer exhibited short-term benefits in terms of reduced surgical interference, earlier recovery of bowel function, improved cosmetic effect, stable immune function and homeostasis. ${ }^{16-18}$ Hisada $\mathrm{M}$ et al ${ }^{19}$ suggested the short-term outcomes of transanal NOSE and conventional laparoscopic-assisted anterior resection for rectal cancer were compared. The average times to the start of oral ingestion were 4 and 4.3 days, respectively, and the hospital stays were 11 and 11.8 days in length, respectively. These results also confirmed that patients experienced a shorter postoperative pain period and less usage of analgesia in the NOSE group. Nishimura $\mathrm{A}$ et $\mathrm{al}^{20}$ revealed that 
Table 4 Local and distant recurrence

\begin{tabular}{|c|c|c|c|c|c|c|c|}
\hline Case & Group & Gender & $\begin{array}{l}\text { Distance of primary } \\
\text { tumor from anal verge } \\
(\mathrm{cm})\end{array}$ & Stage & $\begin{array}{l}\text { Time of } \\
\text { recurrence }\end{array}$ & Site of recurrence & $\begin{array}{l}\text { Treatment after } \\
\text { recurrence }\end{array}$ \\
\hline 1 & NOSE & $\mathrm{F}$ & 25 & T3NOMO & 23 months & Anastomosis & Surgery \\
\hline 2 & NOSE & M & 10 & T3N2M0 & 13 months & Lung & Chemotherapy \\
\hline 3 & NOSE & M & 10 & T3N2M0 & 18 months & Liver & Radiofrequency ablation \\
\hline 4 & NOSE & $\mathrm{F}$ & 26 & T3NOMO & 13 months & Local lymph node & Surgery \\
\hline 5 & NOSE & M & 9 & T3N2M0 & 6 months & Liver & Surgery \\
\hline 6 & NOSE & $\mathrm{F}$ & 20 & T3N2M0 & 36 months & Lung & Chemotherapy \\
\hline 7 & NOSE & M & 18 & T3N2M0 & 24 months & Liver & Chemotherapy \\
\hline 8 & NOSE & M & 12 & T3N2M0 & 5 months & Liver and lung & Chemotherapy \\
\hline 9 & LAP & M & 25 & T4NIMO & 8 months & Liver & Chemotherapy \\
\hline 10 & LAP & M & 20 & T3N2M0 & 6 months & Liver & Chemotherapy \\
\hline II & LAP & M & 15 & T3N2M0 & 18 months & Liver & Chemotherapy \\
\hline 12 & LAP & $\mathrm{F}$ & 10 & T3N2M0 & 12 months & Liver & Surgery \\
\hline 13 & LAP & $\mathrm{F}$ & 8 & T3N2M0 & 25 months & Abdominal pelvic cavity & Chemotherapy \\
\hline 14 & LAP & $\mathrm{F}$ & 10 & T3N2M0 & 12 months & Lung & Chemotherapy \\
\hline 15 & LAP & M & 12 & T3NOMO & 13 months & Liver & Surgery \\
\hline
\end{tabular}

Note: NOSE, totally laparoscopic anterior resection with transanal specimen extraction.

Abbreviation: LAP, laparoscopic anterior resection with minilaparotomy.

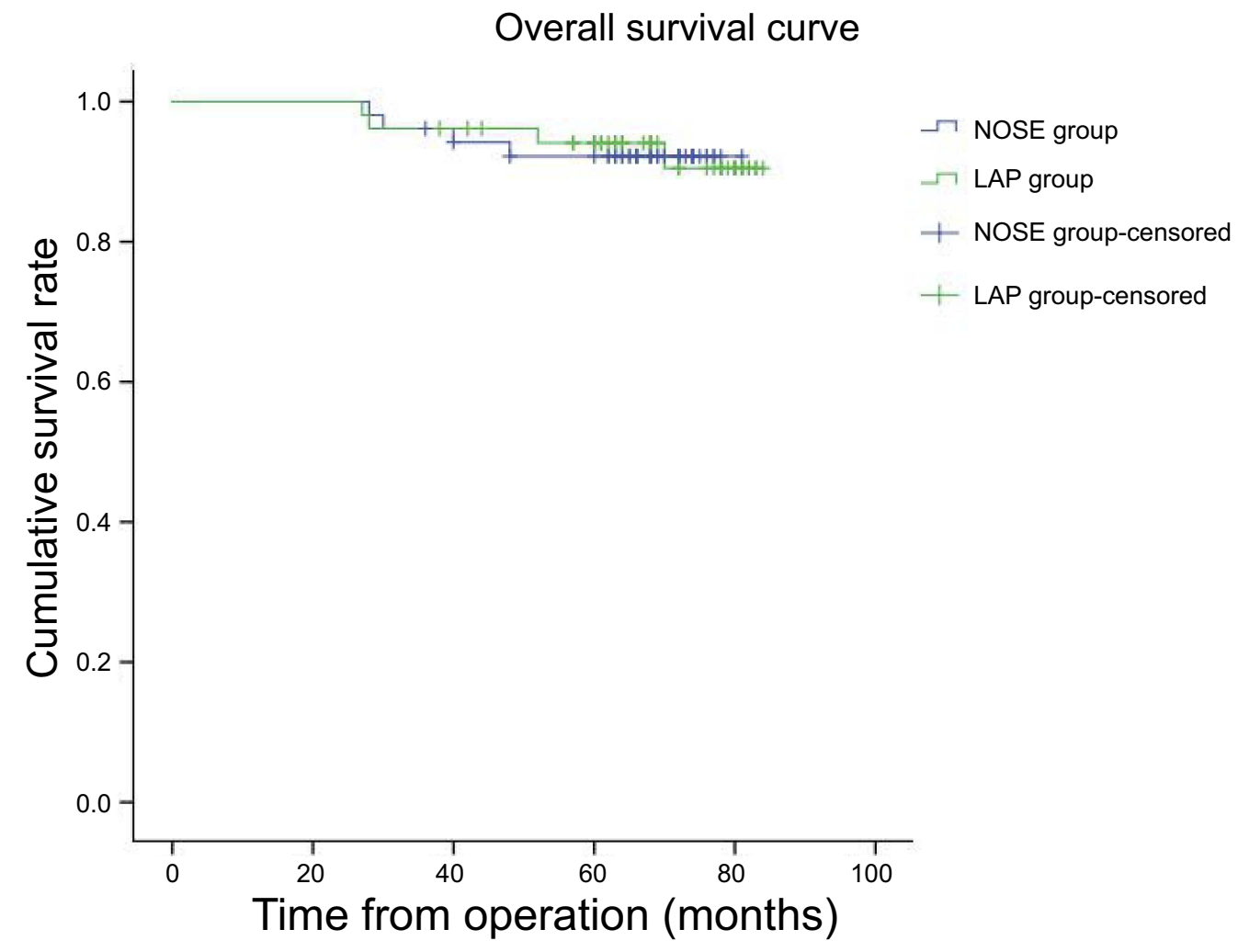

Figure I The overall survival curve clears that 5-year overall survival rate in the NOSE group and LAP group were $92.3 \%$ and $94.2 \%$, respectively. There was no significant difference between the NOSE and LAP groups $(p=0.985)$. NOSE, totally laparoscopic anterior resection with transanal specimen extraction; LAP laparoscopic anterior resection with minilaparotomy.

all patients who underwent NOSE in his study were able to walk at postoperative day 1 and had a significant advantage of faster recovery of intestinal function. Consistent with this study, our study also found that the time to first flatus of patients in the NOSE group was significantly earlier compared with the LAP group $(2.8 \pm 0.8$ vs 3.2 


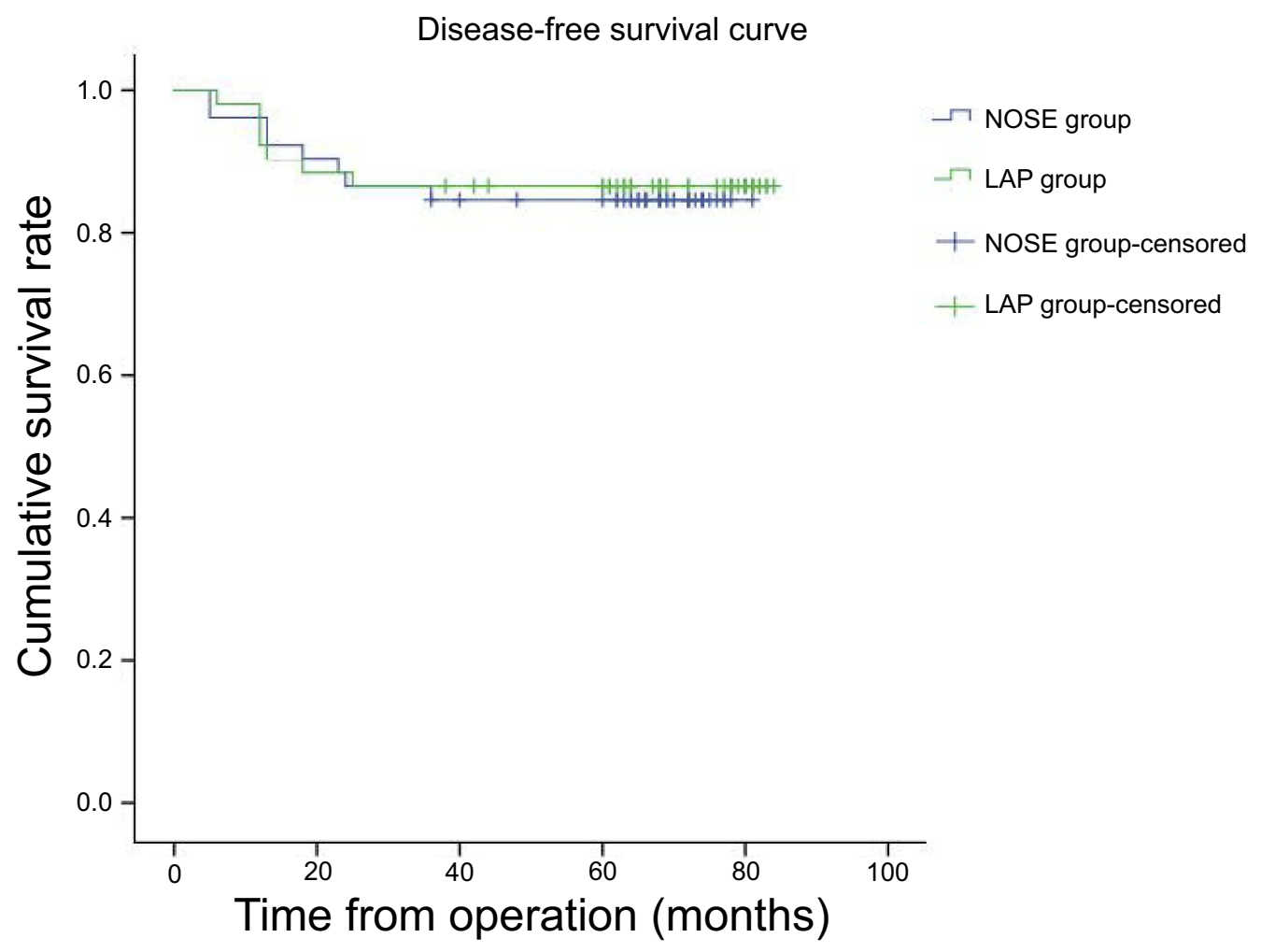

Figure 2 The disease-free survival curve clears that 5-year disease-free survival rate in the NOSE group and LAP group were $84.6 \%$ and $86.5 \%$, respectively. There was no significant difference between the NOSE and LAP groups $(p=0.802)$. NOSE, totally laparoscopic anterior resection with transanal specimen extraction; LAP laparoscopic anterior resection with minilaparotomy.

\pm 0.9 days; $p=0.042$ ). Moreover, compared with patients in the LAP group, patients in the NOSE group had lower postoperative pain on POD1 and less analgesia required $(2.5 \%$ vs $7.7 \%, p=0.032)$. This finding may be because complete laparoscopy reduces the chance of the intraabdominal organs making contact with the external environment, so gastrointestinal function is less disturbed. ${ }^{21}$ In addition, given the absence of abdominal incisions, patients do not limit their early functional activities due to incision pain after surgery, thereby further promoting the recovery of gastrointestinal function.

Naturally, opening of the rectal stump and delivery of the proximal colon and the anvil via the anorectum raises concerns for infection control. Costantino FA et $\mathrm{al}^{22}$ prospectively assessed the peritoneal contamination after NOSE surgery, and $100 \%$ of sample fluid cultures were positive. These authors found that although contamination invariably occurred, it did not translate into infectious morbidity, and no significant differences in clinical outcomes relative to those of the standard laparoscopic approach were noted. Similarly, the NOSE group in our series presented a relatively higher rate of leucocytosis and a statistically higher level of CPR at the POD1 evaluation, and these findings may reflect peritoneal contamination during the operation. However, it is noteworthy that the complications of the two groups were not significantly different. Thus, we believe that the risk of contamination can be minimized through distal rectal irrigation before delivering the proximal colon via the rectum.

Another possible concern in this described approach is the potential risk of cancer cell exfoliation, implantation and local recurrence at the rectal stump opening. The fullness the principles of oncologic surgery is the most important factor for the treatment of cancer regardless of the approach. In the NOSE group, no added technical difficulty was noted for maintaining the oncological principle, in particular during high ligation of inferior mesenteric artery and bowel mobilization, by virtue of the standard multiport system. Hence, there was no difference in oncological outcomes between the two groups in this study. No gross or microscopic positive resection margins were observed in either group. Furthermore, adequate lymph node dissection is an important factor affecting the long-term prognosis of patients with colorectal cancer. ${ }^{23}$ Our results showed that the average number of harvested lymph nodes was 19.1 in the NOSE group, which exceeded the acceptable 12 nodes. 
Few reports comparing NOSE and conventional laparoscopic surgery for colorectal cancer have reported long-time survival data. Kim et $\mathrm{al}^{12}$ reported on 58 patients who underwent total laparoscopic anterior resection with transvaginal specimen extraction, and they found no difference in 3-year disease-free survivals between the NOSE group and the conventional LAP group. Meanwhile, the NOSE group experienced no port-site or transvaginal access-site recurrence. Similarly, Park JS et $\mathrm{al}^{13}$ conducted a case control study comparing the clinical outcomes of 34 patients who underwent total laparoscopic hemicolectomy with NOSE with same number of patients who underwent a conventional laparoscopically assisted approach. These authors suggested that there was no transvaginal accesssite recurrence or posterior colpotomy-related complications after a median follow-up of 23 months. In this study, two patients developed local recurrences in the NOSE group, including one case of anastomosis recurrence and another case of local lymph node metastasis. Anastomotic recurrence should be associated with tumor cell implantation following the reversed flow of flushing fluid. We used the oesophageal band to close the bowel in the first 8 patients, but this band is occasionally loosened. Thus, we changed a 1-0 loop needle to tighten the bondage and avoid reversed flow. In the other patients, 17 lymph nodes were harvested, and none of these nodes harbored a metastasis. We think that the lymph node metastasis stage was avoided in this patient. During the mesenteric division of the sigmoid, care was taken to sufficiently open the window into the mesentery to optimize left colon mobilization and to gain sufficient lymph node dissection while avoiding injury of the marginal colonic artery.

The present study is limited by its retrospective nature and its inherent selection bias. To minimize selection bias, cases were matched to controls according to location of the tumor and other clinical characteristics. The second limitation is that the study excluded bulky tumors and obese patients to avoid damage to anal sphincter function during specimen retrieval. The size of the tumor as well as the mesorectum with relation to the size of the anal canal are import clinical factors determining the success of this method. In this study, the specimen was unable to be delivered through the anus in three patients given the two reasons mentioned above. Finally, the statistical power is insufficient for assessment of oncological outcomes because the sample size is small.

\section{Conclusion}

We have successfully performed total laparoscopic anterior resection with intracorporeal anastomosis and transanal specimen extraction in 52 colorectal cancer patients. This method can achieve minimal invasiveness and has more advantages, including less pain, less tissue trauma and faster recovery of intestinal function. Furthermore, NOSE surgery does not increase the risk of local recurrence, especially transanal access-site or port-site recurrence, and its long-term survival outcomes are basically the same as conventional laparoscopic surgery. Although a learning curve exists for NOSE surgery, it does not require any special skill or equipment. As the concept of this procedure proposed in this study continues to spread, we believe it may be widely accepted.

\section{Acknowledgments}

This work was supported by the Beijing Hope Run Special Fund of Cancer Foundation of China (LC2017A19) and the Capital's Funds for Health Improvement and Research (2016-2-4022).

\section{Disclosure}

The authors report no conflicts of interest in this work.

\section{References}

1. Athanasiou CD, Robinson J, Yiasemidou M, Lockwood S, Markides GA. Laparoscopic vs open approach for transverse colon cancer. A systematic review and meta-analysis of short and long term outcomes. Int J Surg. 2017;41:78-85. doi:10.1016/j.ijsu.2017.03.050

2. Reza MM, Blasco JA, Andradas E, Cantero R, Mayol J. Systematic review of laparoscopic versus open surgery for colorectal cancer. $\mathrm{Br} \mathrm{J}$ Surg. 2006;93:921-928. doi:10.1002/bjs.5430

3. Franklin ME Jr, Liang S, Russek K. Natural orifice specimen extraction in laparoscopic colorectal surgery: transanal and transvaginal approaches. Tech Coloproctol. 2013;17(Suppl 1):S63-S67. doi:10.1007/s10151-0120938-y

4. Akamatsu H, Omori T, Oyama T, et al. Totally laparoscopic sigmoid colectomy: a simple and safe technique for intracorporeal anastomosis. Surg Endosc. 2009;23:2605-2609. doi:10.1007/s00464-009-0406-6

5. Awad ZT, Qureshi I, Seibel B, Sharma S, Dobbertien MA. Laparoscopic right hemicolectomy with transvaginal colon extraction using a laparoscopic posterior colpotomy: a 2-year series from a single institution. Surg Laparosc Endosc Percutan Tech. 2011;21:403-408. doi:10.1097/SLE.0b013e31823945ac

6. Gettman MT, Lotan Y, Napper CA, Cadeddu JA. Trans-vaginal laparoscopic nephrectomy: development and feasibility in the porcine model. Urology. 2002;59:446-450.

7. Kalloo AN, Singh VK, Jagannath SB, et al. Flexible transgastric peritoneoscopy: a novel approach to diagnostic and therapeutic interventions in the peritoneal cavity. Gastrointest Endosc. 2004;60:114-117.

8. Franklin ME Jr, Ramos R, Rosenthal D, Schuessler W. Laparoscopic colonic procedures. World J Surg. 1993;17:51-56.

9. Cheung HY, Leung AL, Chung CC, Ng DCK, Li MKW. Endo-laparoscopic colectomy without mini-laparotomy for left-sided colonic tumors World J Surg. 2009;33:1287-1291. doi:10.1007/s00268-009-0006-6 
10. Saad S, Hosogi H. Natural orifice specimen extraction for avoiding laparotomy in laparoscopic left colon resections: a new approach using the McCartney tube and the tilt top anvil technique. $J$ Laparoendosc Adv Surg Tech A. 2010;20:689-692. doi:10.1089/ lap.2010.0303

11. Sylla P, Bordeianou LG, Berger D, et al. A pilot study of natural orifice transanal endoscopic total mesorectal excision with laparoscopic assistance for rectal cancer. Surg Endosc. 2013;27:3396-3405. doi:10.1007/s00464-013-2922-7

12. Kim HJ, Choi GS, Park JS, Park SY, Ryuk JP, Yoon SH. Transvaginal specimen extraction versus conventional minilaparotomy after laparoscopic anterior resection for colorectal cancer: mid-term results of a case-matched study. Surg Endosc. 2014;28:2342-2348. doi:10.1007/s00464-014-3466-1

13. Park JS, Choi GS, Kim HJ, Park SY, Jun SH. Natural orifice specimen extraction versus conventional laparoscopically assisted right hemicolectomy. Br J Surg. 2011;98:710-715. doi:10.1002/bjs.7419

14. Xingmao Z, Haitao Z, Jianwei L, Huirong H, Junjie H, Zhixiang Z. Totally laparoscopic resection with natural orifice specimen extraction (NOSE) has more advantages comparing with laparoscopicassisted resection for selected patients with sigmoid colon or rectal cancer. Int J Colorectal Dis. 2014;29:1119-1124. doi:10.1007/ s00384-014-1950-7

15. Zhang $\mathrm{X}$, Zhou $\mathrm{H}$, Hou $\mathrm{H}$, Hu J, Wang $\mathrm{H}$, Zhou Z. Totally laparoscopic resection with natural orifice specimen extraction for carcinoma of sigmoid colon and rectum: a feasible and innovative technique. J Clin Gastroenterol. 2014. doi:10.1097/ MCG.0000000000000038

16. Franklin ME Jr, Liang S, Russek K. Integration of transanal specimen extraction into laparoscopic anterior resection with total mesorectal excision for rectal cancer: a consecutive series of 179 patients. Surg Endosc. 2013;27:127-132. doi:10.1007/s00464-012-2440-z
17. D'Hoore A, Wolthuis AM. Laparoscopiclowanteriorresection and transanal pull-through for low rectal cancer: a Natural Orifice Specimen Extraction (NOSE) technique. Colorectal Dis. 2011;13:28-31. doi:10.1111/j.1463-1318.2011.02773.x

18. Bie M, Wei ZQ. A new colorectal/coloanal anastomotic technique in sphincter-preserving operation for lower rectal carcinoma using transanal pull-through combined with single stapling technique. Int $J$ Colorectal Dis. 2013;28:1517-1522. doi:10.1007/s00384-013-1723-8

19. Hisada M, Katsumata K, Ishizaki T, et al. Complete laparoscopic resection of the rectum using natural orifice specimen extraction. World J Gastroenterol. 2014;20:16707-16713. doi:10.3748/wjg.v20. i44.16707

20. Nishimura A, Kawahara M, Suda K, Makino S, Kawachi Y, Nikkuni K. Totally laparoscopic sigmoid colectomy with transanal specimen extraction. Surg Endosc. 2011;25:3459-3463. doi:10.1007/s00464011-1716-z

21. Akiyoshi T, Kuroyanagi H, Oya M, et al. Short-term outcomes of laparoscopic rectal surgery for primary rectal cancer in elderly patients: is it safe and beneficial? $J$ Gastrointest Surg. 2009;13:1614-1618. doi:10.1007/s11605-009-0961-0

22. Costantino FA, Diana M, Wall J, Leroy J, Mutter D, Marescaux J. Prospective evaluation of peritoneal fluid contamination following transabdominal vs. transanal specimen extraction in laparoscopic left-sided colorectal resections. Surg Endosc. 2012;26:1495-1500. doi:10.1007/s00464-011-2066-6

23. Yamamoto S, Inomata M, Katayama H, et al. Japan clinical oncology group colorectal cancer study group. short- term surgical outcomes from a randomized controlled trial to evaluate laparoscopic and open D3 dissection for stage II/III colon cancer: Japan Clinical Oncology Group Study JCOG 0404. Ann Surg. 2014;260:23-30. doi:10.1097/ SLA.0000000000000499

\section{Publish your work in this journal}

Cancer Management and Research is an international, peer-reviewed open access journal focusing on cancer research and the optimal use of preventative and integrated treatment interventions to achieve improved outcomes, enhanced survival and quality of life for the cancer patient.
The manuscript management system is completely online and includes a very quick and fair peer-review system, which is all easy to use. Visit http://www.dovepress.com/testimonials.php to read real quotes from published authors 